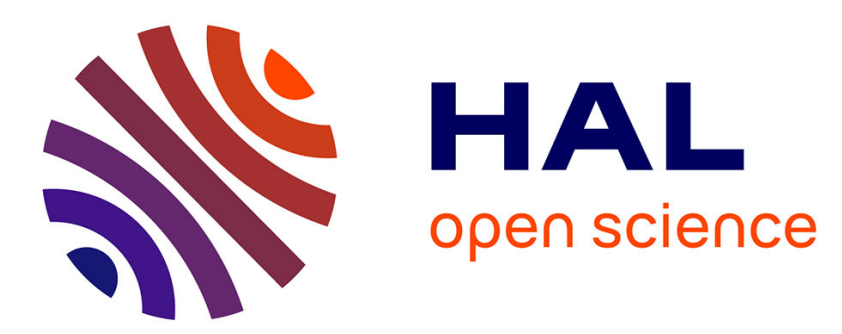

\title{
Observation of nonlinear interactions in large-scale density enhancements of the high-latitude ionosphere
}

Arnaud Masson, Franqois Lefeuvre, Zheng y Zhao, Dominique Lagoutte, Jean-Louis Rauch

\section{- To cite this version:}

Arnaud Masson, Franqois Lefeuvre, Zheng y Zhao, Dominique Lagoutte, Jean-Louis Rauch. Observation of nonlinear interactions in large-scale density enhancements of the high-latitude ionosphere. Journal of Geophysical Research Space Physics, 1999, 104 (A10), pp.22499-22521. 10.1029/1999JA900200 . insu-02879940

HAL Id: insu-02879940

https://hal-insu.archives-ouvertes.fr/insu-02879940

Submitted on 24 Jun 2020

HAL is a multi-disciplinary open access archive for the deposit and dissemination of scientific research documents, whether they are published or not. The documents may come from teaching and research institutions in France or abroad, or from public or private research centers.
L'archive ouverte pluridisciplinaire HAL, est destinée au dépôt et à la diffusion de documents scientifiques de niveau recherche, publiés ou non, émanant des établissements d'enseignement et de recherche français ou étrangers, des laboratoires publics ou privés. 


\title{
Observation of nonlinear interactions in large-scale density enhancements of the high-latitude ionosphere
}

\author{
Arnaud Masson, ${ }^{1}$ François Lefeuvre, Zheng Y. Zhao, ${ }^{2}$ Dominique Lagoutte, \\ and Jean-Louis Rauch \\ Laboratoire de Physique et Chimie de l'Environnement, CNRS, Orléans, France
}

\begin{abstract}
Simultaneous measurements of electron density and ELF electric field by the AUREOL-3 satellite have allowed Cerisier et al. [1985] to point out the existence of large-scale density irregularities in the high-latitude topside $F$ region and to establish the role of the gradient drift instability in the plasma structuring. High-resolution spectra of the density and field fluctuations show the presence of low-frequency peaks linked by relationships such that $f_{m}+f_{n}=f_{m+n}$. Bispectral analyses point out nonlinear interactions between waves at 7.8 and $15.6 \mathrm{~Hz}$ and density irregularities at $5.85 \mathrm{~Hz}$. The consistency of the results with regard to instability models is discussed. The existence of an interaction process involving Schumann resonances is suggested.
\end{abstract}

\section{Introduction}

Large-scale (tens of kilometers) density irregularities are currently observed in the high-latitude $F$ region of the ionosphere and above [Vickrey et al., 1980; Muldrew and Vickrey, 1982; Rino et al., 1983; Béghin et al., 1985; Weber et al., 1984, 1985, 1986; Kersley et al., 1988; Basu et al., 1988, 1990]. It has already been shown that they are the seat of gradient drift instabilities [Linson and Workman, 1970; Muldrew and Vickrey, 1982; Cerisier et al., 1985; Basu et al., 1990]. The object of the present paper is to point out nonlinear interactions associated with these plasma inhomogeneities.

Simultaneous density and electric field fluctuations, recorded on board the AUREOL-3 satellite in the highlatitude $F$ region, have been analyzed by Cerisier et al. [1985]. One of the main results was that instabilities are developed on the positive gradients of large-scale density irregularities convected by the DC electric field. As the same spectral indices were found for the density and electric field fluctuations, the authors concluded that the instabilities were of the gradient drift type.

However, irregularity and field spectra often present oscillations at the smallest frequencies, i.e., at the longest wavelengths (at least in the zero phase velocity approximation). If most of them can be approximated

\footnotetext{
${ }^{1}$ Now at Swedish Institute of Space Physics, Uppsala. China.

${ }^{2}$ On leave from Department of Space Physics, Wuhan University,
}

Copyright 1999 by the American Geophysical Union.

Paper number 1999JA900200.

0148-0227/99/1999JA900200\$09.00 by a single spectral index, two power law segments, having different indices, may be required to obtain a correct fit. Villain et al. [1986] found that $25 \%$ of AUREOL-3 irregularity spectra are better fitted by twocomponent spectra. Proposed interpretations were discussed by Tsunoda [1988]. To study that point, highresolution spectral analyses have been performed by Zhao et al. [1997] on AUREOL-3 data already analyzed by Cerisier et al. [1985]. They show the existence of well-defined frequency peaks at the smallest frequencies both on the irregularities and on the field spectra. As frequency relationships of the type $f_{m}+f_{n}=f_{m+n}$ are pointed out, it seems reasonable to look for the existence of nonlinear interactions.

Now, the power spectra are second-order statistics. Frequency relationships derived from them may be pure coincidences. One way to check the physical meaning of relationships of the type $f_{m}+f_{n}=f_{m+n}$ is to estimate third-order statistics, i.e., bispectra and bicoherency functions [Kim and Powers, 1979; Lagoutte et al., 1989]. Although nonzero values only point out phase relationships (or phase coupling) they may be interpreted as a signature of a three-wave nonlinear interaction. Applications to space plasmas have been published by Lee and Kuo [1981], Riggin and Kelley [1982], Tanaka et al. [1987], Lagoutte et al. [1989], Trakhtengerts and Hayakawa [1993], Dudok de Wit and Krasnosel'skikh [1995] and Rezeau et al. [1997].

It is the aim of the present paper to revisit Aureol-3 density and electric field fluctuations already analyzed by Cerisier et al. [1985], in order to (1) determine the frequency peaks in the power spectra with the maximum possible accuracy, (2) show the power variations when the satellite crosses the density inhomogeneities, and (3) identify any eventual nonlinear process of 

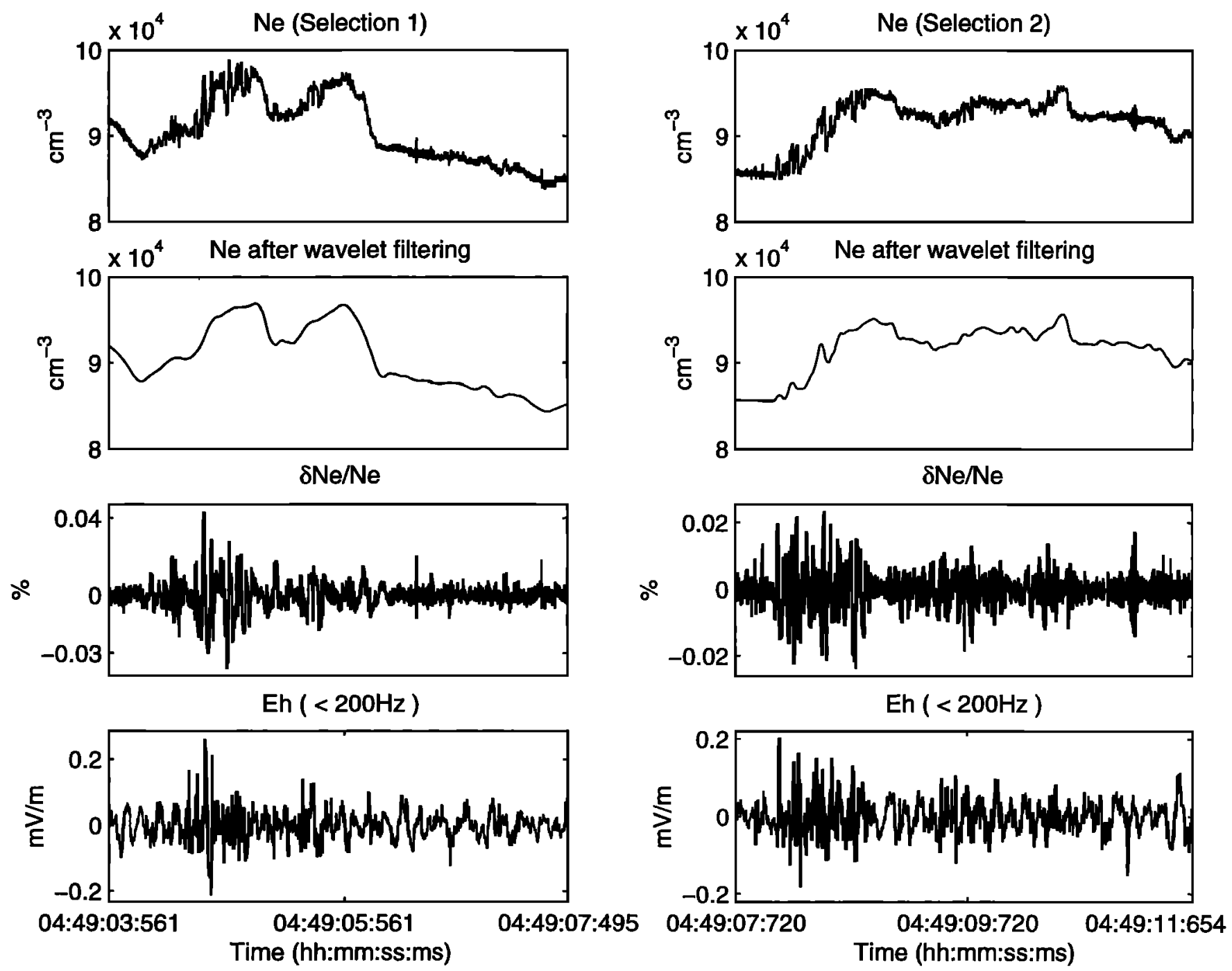

Figure 1. Aureol-3 data to be analyzed (see text): left the first time interval (or selection 1), and right the second (selection 2).

energy transfer between the fluctuations and the frequencies (or wavelengths).

\section{Data}

The data studied by Cerisier et al. [1985] have been obtained by the low-altitude (400-2000 km) Aureol-3 satellite launched into a nearly polar orbit on September 21,1981 . The satellite was three axis stabilized. Low-energy electrons and ions were measured by the SPECTRO experiment [Bosqued et al., 1982]. The electron density was measured by the high-resolution Interferometer Self-Oscillating Probe (ISOPROBE) mutual impedance sounder [Béghin et al., 1982]. In the mode of operation used at the time of the recording, the electron density value $\left(N_{e}\right)$ was estimated every 0.2 ms. The wave field measurements were performed by the Très Basses Fréquences (TBF) experiment [Berthelier et al., 1982]. Waveforms associated with the measurements of two electric and three magnetic wave field components in the ELF frequency range (1-1500 $\mathrm{Hz}$ ) were transmitted to the ground. According to the characteristics of the filters, the corresponding time series were also sampled at $5 \mathrm{kHz}$. In what follows, we shall mainly consider the $E_{h}$ electric component that stays approximately perpendicular to the Earth's magnetic field direction.

Detailed analyses of $N_{e}(\mathrm{t})$ and $E_{h}(t)$ fluctuations have been performed over time intervals where medium scale irregularities in electron density are identified. Here, the presentation will focus on TBF and ISOPROBE data for which Cerisier et al. [1985] have made a spectral analysis. They were recorded on March 16,1982 , during a crossing of a diffuse aurora region (precipitated electrons are seen on SPECTRO between $\sim 0447: 30$ and $\sim 0451: 00 \mathrm{UT})$. At the time of the analysis, the satellite was approximately at $650 \mathrm{~km}$ altitude. The invariant latitude was $\sim 70^{\circ}(\mathrm{L} \sim 8.8)$ and 
the Magnetic Local Time (MLT) was 0744. There are $8 \mathrm{~s}$ of data that can be analyzed. For technical reasons, they have been split into two 4-s time intervals. From hereinafter, they will be referred as selection 1 and selection 2.

The top panels of Figure 1 represent the $N_{e}(t)$ time series. The electron density fluctuations $\delta N_{e}(t) / N_{e}(t)$ are obtained here by applying a wavelet decomposition at level 8 based on the Daubechies 5 wavelet [Daubechies, 1992]. This allows to remove discontinuities we get when subtracting mean values as of Cerisier et al. [1985] but the results are basically the same. If we denote by $N_{e}^{0}(t)$ the level 8 approximation obtained after wavelet filtering of the $N_{e}(t)$ time series (see second panels of Figure 1), the expression of the electron density fluctuations is given by

$$
\frac{\delta N_{e}(t)}{N_{e}(t)}=\frac{N_{e}(t)-N_{e}^{0}(t)}{N_{e}(t)}
$$

To be consistent with the wave data (see below), they are passed through a $200-\mathrm{Hz}$ low-pass filter. The lowfrequency parts of the $\delta N_{e}(t) / N_{e}(t)$ time series are given in the third panels of Figure 1.

The original waveform of the electric field component is not represented here. A hiss emission, with a low cutoff frequency around $300 \mathrm{~Hz}$, masks the phenomenon we are interested in. It is removed by filtering the $E_{h}(t)$ time series below $200 \mathrm{~Hz}$. The final time series are displayed in the bottom panels of Figure 1. Strong amplitude variations are seen both in $E_{h}(t)$ and in $\delta N_{e}(t) / N_{e}(t)$ during the time intervals: 0449:04.161-0449:04.961 UT and 0449:08.0400449:08.840 UT. In order to extract physical information from these strong inhomogeneities, spectral analysis has been performed.

\section{Spectral Analysis}

The estimation of the autopower spectrum of a time series requires the stationarity condition, that is to say the conservation of its moments through time (at least over the time of observation). In our case, the temporal evolution of the moments reveals a clear departure from stationarity (see Figure 2 of Zhao et al. [1997]). Nevertheless, discrete events, or transients, seem to be superimposed on random fluctuations. Following Hinich [1990] and Pflug et al. [1995], we will assume that a transient signal has the characteristics of a deterministic signal. Furthermore, for the sake of simplicity, we will consider the noise to be normally distributed.

Let $x(t)$ be the time series associated with one of the fluctuations. According to our model, it can be written

$$
x(t)=d(t)+n(t)
$$

where $d(t)$ and $n(t)$ label the transient signal and the noise.
Assuming $n(t)$ to be stationary over the time of observation, the $x(t)$ autopower spectrum can be written

$$
S_{x}(f)=E\left[X^{*}(f) X(f)\right]
$$

with $X(f)$ the Fourier transform of $x(t), X^{*}(f)$ its complex conjugate, and $E[]$ the mathematical expectation.

Then, assuming $d(t)$ and $n(t)$ uncorrelated, we get

$$
S_{x}(f)=S_{d}(f)+S_{n}(f)
$$

with $D(f)$ and $N(f)$ the Fourier transform of $d(t)$ and $n(t)$ respectively, $D^{*}(f)$ and $N^{*}(f)$ their complex conjugate and $S_{d}(f)=D(f) D^{*}(f)$ and $S_{n}(f)=$ $E\left[N(f) N^{*}(f)\right]$. The stationarity condition a priori required on $x(t)$ is now only needed on $n(t)$.

Estimations of the autopower spectra of $E_{h}(t)$ and $\delta N_{e}(t) / N_{e}(t)$ are performed using the Welch [1967] periodogram method over the full 4-s time intervals (13 FFT are used, there is a $50 \%$ overlapping). The frequency resolution is $\pm 0.97 \mathrm{~Hz}$. The results are displayed in Figure 2.

Let first consider the autopower spectra of $E_{h}$ (Figure 2, top plots). Comparing the two analyses, one observes a well-defined peak at $7.8 \mathrm{~Hz}$, more or less stable peaks around $15.6 \mathrm{~Hz}, 21$ to $24 \mathrm{~Hz}$, and $31.2 \mathrm{~Hz}$, then a time-dependent peak $(11.7 \mathrm{~Hz})$. For the main peak, the frequency resolution may be improved by integrating over the two time series. One obtains $7.8 \pm 0.4 \mathrm{~Hz}$. As far as the approximately stable secondary peaks are concerned, the absolute uncertainty on the frequencies of the peaks is $2 \mathrm{~Hz}$.

Similar phenomena are observed on the autopower spectra of $\delta N_{e} / N_{e}$ (Figure 2, bottom plots). The peaks at $5.85,13.65$, and $17.55 \mathrm{~Hz}$ are approximately stable. The peak at $21.45 \mathrm{~Hz}$ is time dependent. Except at $11.7 \mathrm{~Hz}$, peaks in the electric field are associated with peaks in the density fluctuations, which is consistent with the properties of a wave in a plasma as derived from the Maxwell's equations. Uncertainties in the frequency values may be due to the generation process or/and to the propagation from the source to the observation point. We will return on this subject later on.

An important point in the analysis is to determine whether the frequency peaks seen on the $E_{h}$ power spectra may be associated with electrostatic or electromagnetic emissions. In order to answer that question autopower spectra of the second electric component (nearly parallel to the direction of the Earth's magnetic field) and of the three magnetic components have been computed (but not displayed). As a result, peaks comparable to the ones of Figure 2 are observed on the electric power spectrum but not on the three magnetic. Now, this does not imply that all the modes are electrostatic. The sensitivity of the magnetic sensors varying as $1 / f$, it is very likely that electromag- 

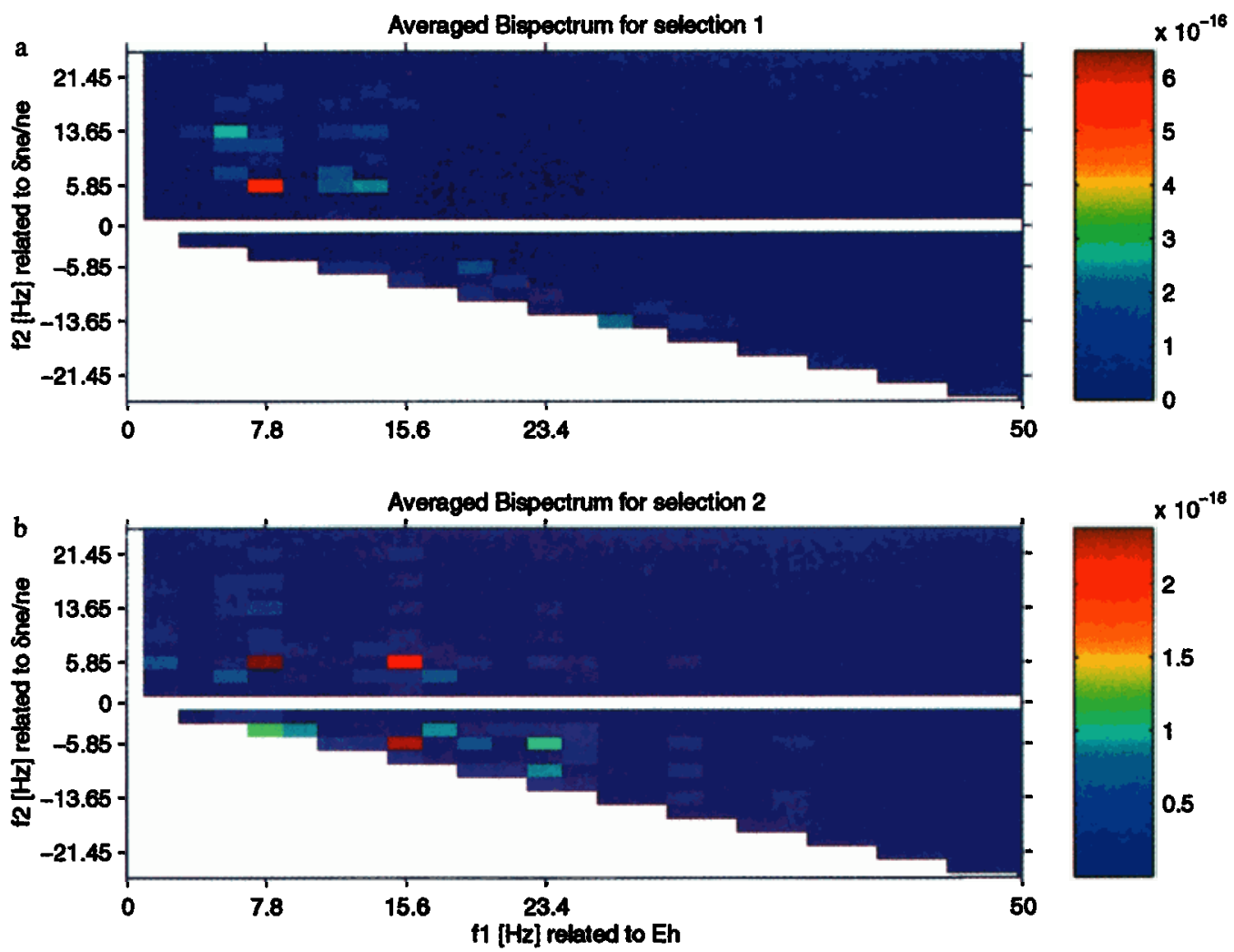

Plate 1. Cross bispectra of the $E_{h}(t)$ and $\delta N_{e}(t) / N_{e}(t)$ time series for (a) selection 1 and (b) selection 2.
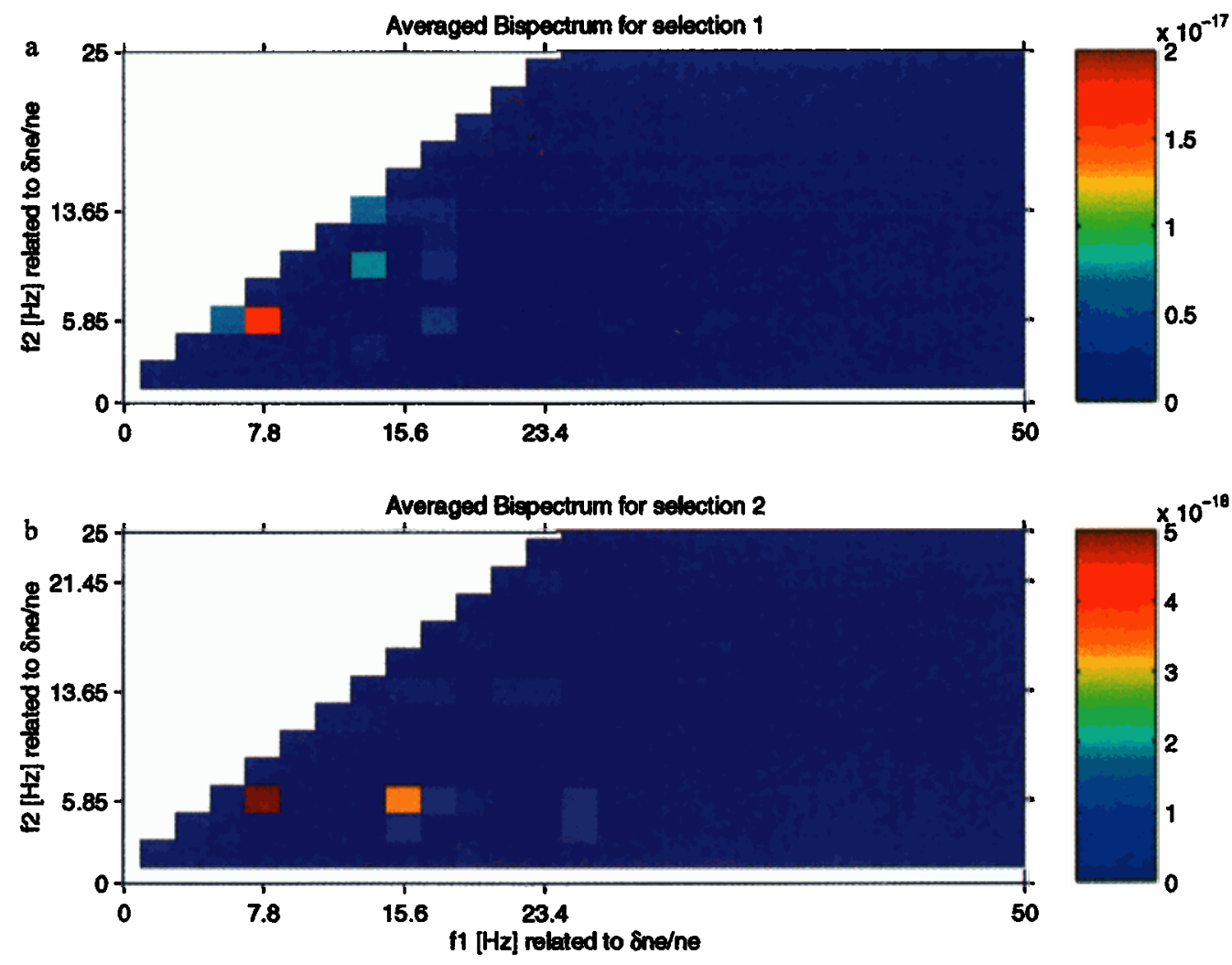

Plate 2. Autobispectra of the $\delta N_{e}(t) / N_{e}(t)$ time series for (a) selection 1 and (b) selection 2. The values which are displayed correspond to autobicoherency values greater than the threshold 0.6 . 
Power Spectra of selection 1

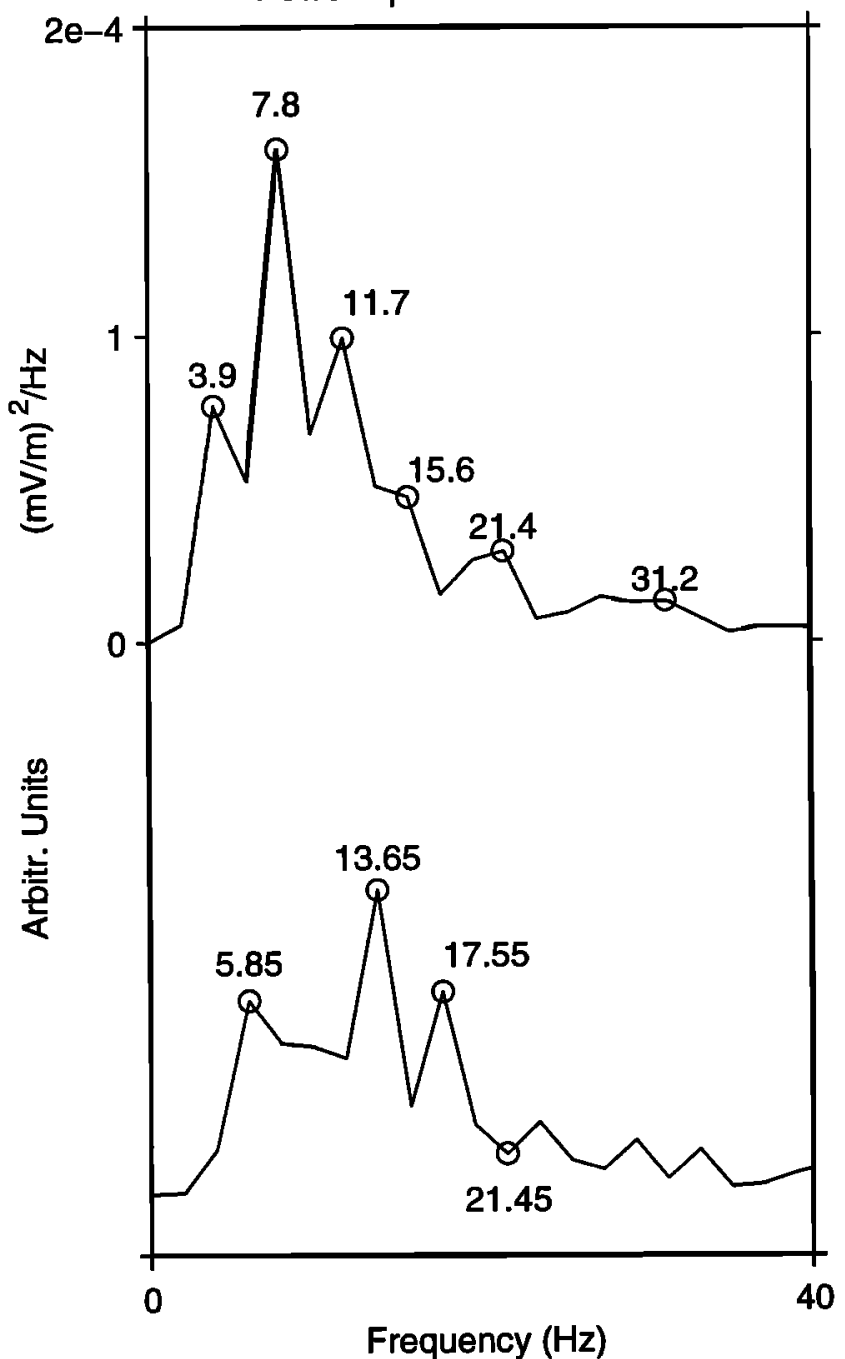

Power Spectra of selection 2

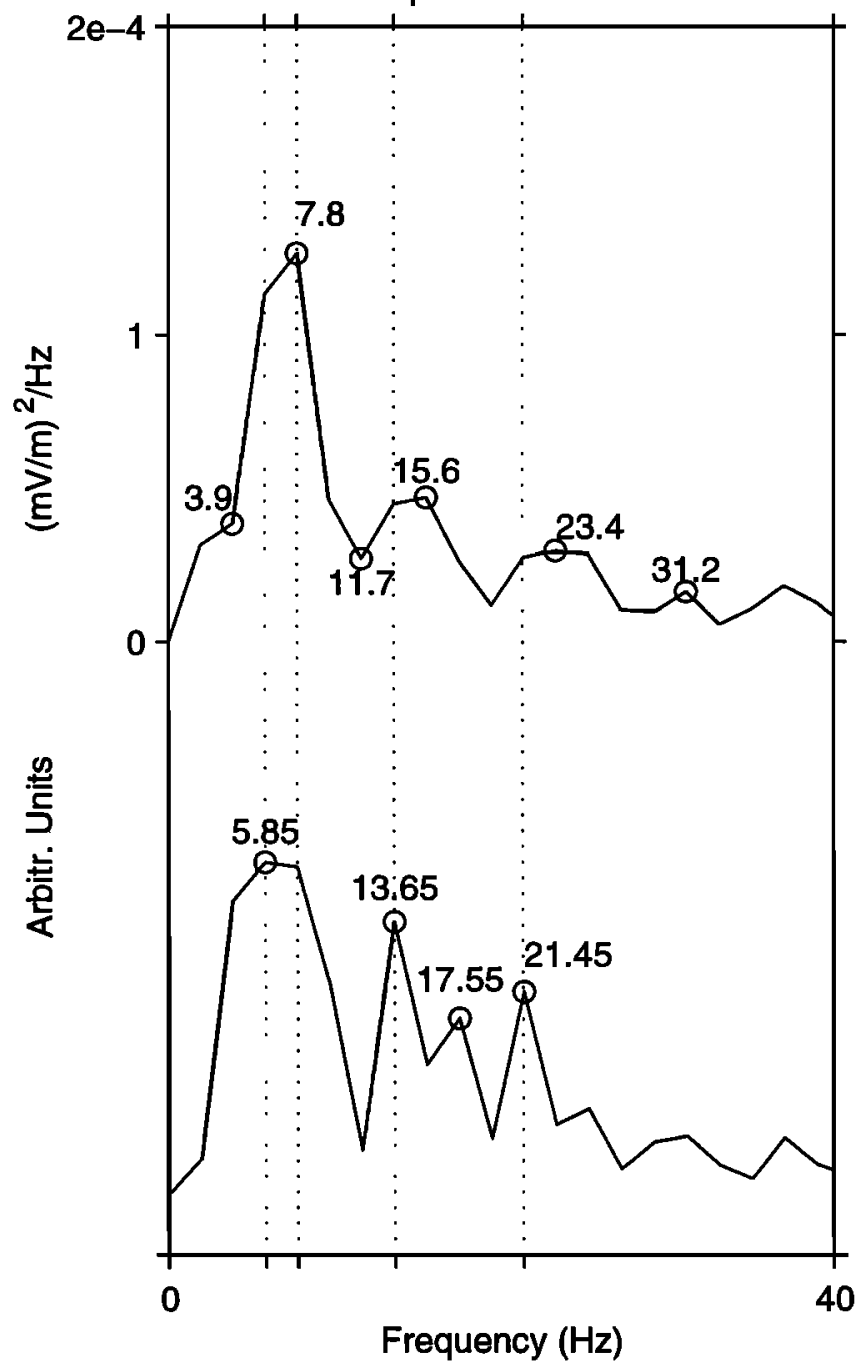

Figure 2. Power spectra: of the $E_{h}$ electric field component (top part of the two panels) and of the fluctuations in electronic density (bottom part of the two panels) for the two time intervals of Figure 1.

netic modes cannot be detected at the lowest frequencies. This is easily checked: (1) assuming cold plasma magnetic modes in a uniform medium, (2) deriving the values of the refractive index $\mathrm{n}$ from the AppletonHartree formula in the case of a parallel propagation [Stix, 1992], then (3) estimating the expected magnetic field power from the relation $n=c B / E_{\perp}$ with $E_{\perp}$ the measured electric component perpendicular to the Earth's magnetic field $B_{0}$ (i.e., $\sim E_{h}$ ). Taking $f=8 \mathrm{~Hz}$, $f_{c e}=1.1 \mathrm{MHz}$ and $f_{p e}=2.8 \mathrm{MHz}$, one obtains $n \cong 400$ for the slow Alfven mode as well as for the fast Alfven mode. Having measured values of $E_{h}$ of the order of $\cong 12 \mu \mathrm{V} / \mathrm{m} / \mathrm{Hz}^{1 / 2}$, one could expect $\cong 15 \mathrm{~m} \gamma / \mathrm{Hz}^{1 / 2}$ on $\mathrm{B}$. Knowing that the sensitivity of the magnetic sensors is of the order of $50 \mathrm{m \gamma} / \mathrm{Hz}^{1 / 2}$ [Berthelier et al., 1982], one concludes that at $8 \mathrm{~Hz}$, peaks on the electric power spectra cannot be seen on the magnetic ones. The hypothesis of an electromagnetic mode cannot be rejected at the lowest frequencies.
Now, a careful examination of Figure 2 shows that frequency relationships such that $f_{m}+f_{n}=f_{m+n}$ exist between the $E_{h}(t)$ and $\delta N_{e}(t) / N_{e}(t)$ peaks. They are summarized below.

For both selections

$$
\begin{aligned}
E_{h}(t)+\delta N_{e}(t) / N_{e}(t) & =\delta N_{e}(t) / N_{e}(t) \\
7.8 \mathrm{~Hz}+5.85 \mathrm{~Hz} & =13.65 \mathrm{~Hz}
\end{aligned}
$$

and for selection 2 only

$$
\begin{aligned}
E_{h}(t)+\delta N_{e}(t) / N_{e}(t) & =\delta N_{e}(t) / N_{e}(t) \\
15.6 \mathrm{~Hz}+5.85 \mathrm{~Hz} & =21.45 \mathrm{~Hz}
\end{aligned}
$$

The temporal evolution of the electric field and the density irregularities power spectra at the frequencies 

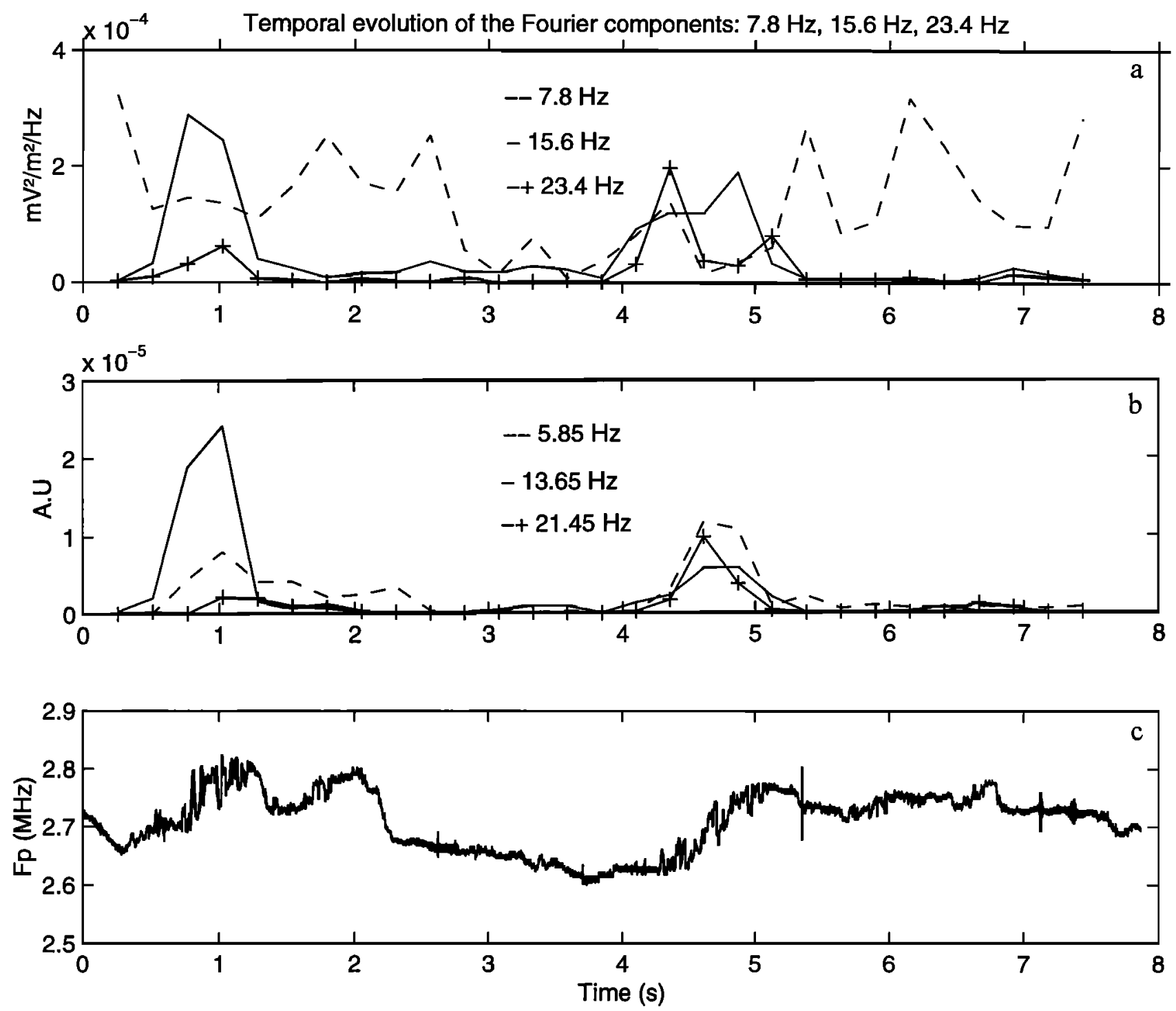

Figure 3. Temporal variation over the full time interval (selection $1+$ selection 2 ) of (a) the values of the power spectrum of the $E_{h}$ field component at $7.8,15.6$, and $23.4 \mathrm{~Hz}$, (b) the values of the power spectrum of the density fluctuations at $5.85,13.65$, and $21.45 \mathrm{~Hz}$, (c) the plasma frequency.

given in (5) and (6) is displayed in Figure 3. The time variation of the plasma frequency is recalled in Figure 3c. The time step is 1282 samples $(0.25 \mathrm{~s})$. At every time step, the power level of a given frequency is obtained from the calculation of an FFT performed over 2564 samples $(0.5 \mathrm{~s})$. Finally, it turns out that the power of the 7.8- $\mathrm{Hz}$ peak on $E_{h}$ (Figure 3a) varies with the large-scale density structures, whereas the power of the two first harmonics $(15.6$ and $23.4 \mathrm{~Hz}$ ) have their maximum values in the regions of positive density gradients. The fact that there is a time delay between the power of the 7.8- $\mathrm{Hz}$ component in the field and the density is consistent with a propagation effect. With regards to the peaks in the irregularity power spectra (Figure 3b), they are all localized inside the density gradients.
The point is to know if the frequency relationships of (5) and (6) are pure coincidences or if they are the result of nonlinear interactions.

\section{Bispectral Analysis}

In order to test the presence of three-wave nonlinear interactions, bispectral analyses are performed. By pointing out phase relationships between Fourier transforms at frequencies such that $f_{m}+f_{n}=f_{m+n}$, potential energy transfers may be identified between frequencies or wavelengths.

The frequency relationships of (5) and (6) clearly suggest to perform a cross-bispectral analysis between $E_{h}(t)$ and $\delta N_{e}(t) / N_{e}(t)$. Additional reasons to make a cross-analysis are first, that the noises from the equip- 
ments are uncorrelated, and second, that this is the only way to involve the frequencies conveying the maximum energy both in $E_{h}(t)$ and in $\delta N_{e}(t) / N_{e}(t)$. Results of autobispectral analyses will be examined later on.

Let $x(t)$ and $y(t)$ be the time series associated with $E_{h}(t)$ and $\delta N_{e}(t) / N_{e}(t)$ respectively, and $X$ and $Y$ their Fourier transforms. The cross-bispectrum of $x(t)$ and $y(t)$ may be written

$$
B\left(f_{m}, f_{n}\right)=E\left[X\left(f_{m}\right) Y\left(f_{n}\right) Y^{*}\left(f_{m+n}\right)\right]
$$

It is equal to zero except for $f_{m}+f_{n}=f_{m+n}$. The stationarity constraint is the same as, if not stronger than, that for the power spectral analysis. Assuming the model of (2) holds, we can write

$$
\begin{aligned}
B\left(f_{m}, f_{n}\right)= & D_{X}\left(f_{m}\right) D_{Y}\left(f_{n}\right) D_{Y}^{*}\left(f_{p}\right)+ \\
& +E\left[N_{X}\left(f_{m}\right) N_{Y}\left(f_{n}\right) N_{Y}^{*}\left(f_{m+n}\right)\right]
\end{aligned}
$$

with $X$ and $Y$ related to $x(t)$ and $y(t)$ as defined for (7).

As a definition, a nonzero value of $B\left(f_{m}, f_{n}\right)$ identifies the presence of a nonlinear phenomenon involving $f_{m}, f_{n}$, and $f_{m+n}$. However, to interpret the results of a bispectral analysis one is lead either to look for relative maxima or to estimate a normalised function $b^{2}\left(f_{m}, f_{n}\right)$ named the bicoherency function. The normalization factor used here involves values of the power spectra at $f_{m}, f_{n}$, and $f_{m+n}$. The bicoherency function varies between 0 and 1 . It measures the degree of phase coupling. Now, considering that a phase coupling is accompanied by an energy transfer when the coupled frequencies convey a sufficient amount of energy only, one is used to estimate the bispectrum first, then to select the values of the peaks for which the bicoherency function get values above a given threshold.

By assuming stationarity for $n_{X}(t)$ and $n_{Y}(t)$, one may estimate the averaged bispectrum over each selection. The estimation is performed using 13 FFTs, each calculated over 2564 samples, i.e., $0.5 \mathrm{~s}$. In so doing, the overlapping is $50 \%$ and the frequency resolution is $\pm 0.97 \mathrm{~Hz}$.

We select the time delays between $x(t)$ and $y(t)$ for which the cross bispectra are maximum. The nonzero parts of the cross bispectra are displayed in Plate 1. The frequency domain is the one defined by Nikias and Petropulu [1993] for cross bispectra.

Let us first examine the cross bispectrum of selection 2 (Plate 1b). The maximum value is obtained for a $X Y Y^{*}$ combination with a time delay $\left(E_{h}(t)\right.$ in advance of $0.36 \mathrm{~s}$ on $\delta N_{e}(t) / N_{e}(t)$. We will propose an interpretation of this value after having examined the results on selection 1 . The top part of the frequency domain (a rectangle) represents the measure of the phase relationships between Fourier components such that $f_{m}+f_{n}=f_{m+n}$, where $f_{m}>0$ and $f_{n}>0$. The lower triangle covers the domain $f_{m}>0, f_{n}<0$, and $f_{m}>2\left|f_{n}\right|$. Two peaks are clearly visible in the upper rectangle: one corresponding to an interaction between the $5.85 \mathrm{~Hz}$ on $\delta N_{e} / N_{e}$ and the $7.8 \mathrm{~Hz}$ on $E_{h}$; and the other between the same $5.85 \mathrm{~Hz}$ on $\delta N_{e} / N_{e}$ and the $15.6 \mathrm{~Hz}$ of $E_{h}$. These results correspond to the expected frequency relations mentioned in (5) and (6). In the lower triangle, a phase relationship is detected between the conjugate of the Fourier component $5.85 \mathrm{~Hz}$ of $\delta N_{e} / N_{e}$ and the $15.6 \mathrm{~Hz} E_{h}$ frequency component according to the relation:

$$
\begin{aligned}
E_{h}(t)-\delta N_{e}(t) / N_{e}(t) & =\delta N_{e}(t) / N_{e}(t) \\
15.6 \mathrm{~Hz}-5.85 \mathrm{~Hz} & =9.75 \mathrm{~Hz}
\end{aligned}
$$

This could be surprising since the $9.75 \mathrm{~Hz}$ does not show up on the $\delta N_{e} / N_{e}$ power spectrum (selection 2) of Figure 2. However, assuming the model of section 2 is valid, one may estimate a power spectrum, from a single FFT, in the supposed region of interactions. This has been done, within a density gradient, i.e., over a time interval where strong transients phenomena are detected. The result is displayed in Figure 4. It shows four well-defined peaks at frequencies: $5.85,9.75,13.65$, and $21.45 \mathrm{~Hz}$, i.e., at the frequencies involved in the quadratic relations (5), (6), and (9). Curiously, the $17.55-\mathrm{Hz}$ peak observed in Figure 2 has disappeared. It is likely that the energy transfers are time dependent. It may be interesting to note that the bicoherency function, estimated over selection 2 , has the same peaks as on the bispectrum; with maximum values greater than 0.6. This means that, independently of the values of the power spectra, there is a strong phase coupling between the frequencies involved.

The cross bispectrum of selection 1 is displayed in Plate 1a. The maximum of the cross bispectrum is found for a $X Y Y^{*}$ combination with a time delay $\left(E_{h}(t)\right.$ in advance of $0.28 \mathrm{~s}$ on $\left.\delta N_{e}(t) / N_{e}(t)\right)$. A single peak emerges. It corresponds to a quadratic interaction between the $7.8-\mathrm{Hz}$ peak in $E_{h}$ and the $5.85-\mathrm{Hz}$ peak in $\delta N_{e}(t) / N_{e}(t)$. The corresponding bicoherency peak is 0.6 .

Let return to the time delays used for selections 1 and 2. They are not surprising. Three non-exclusive explanations may be suggested. First, the phase relationships we are looking for may be modified by frequency shifts $\Delta f_{i}$ associated with a strong turbulence or/and a Doppler effect. In such a case, a time delay is used as a correcting factor. Second, the observation may be taken out of the interaction region. In that case, differences in the propagation characteristics of the two fluctuations generate a time delay. Third, the energy transfer may be time dependent. As an example, a mode may need to reach sufficient amplitude before emptying its energy into one or two other modes. Now, with the few data we cannot go further on.

In order to try to point out the origin of the nonlinearities, autobispectral analyses have been 


\section{Power Spectrum of $\delta$ ne/ne (selection2) inside density enhancement}

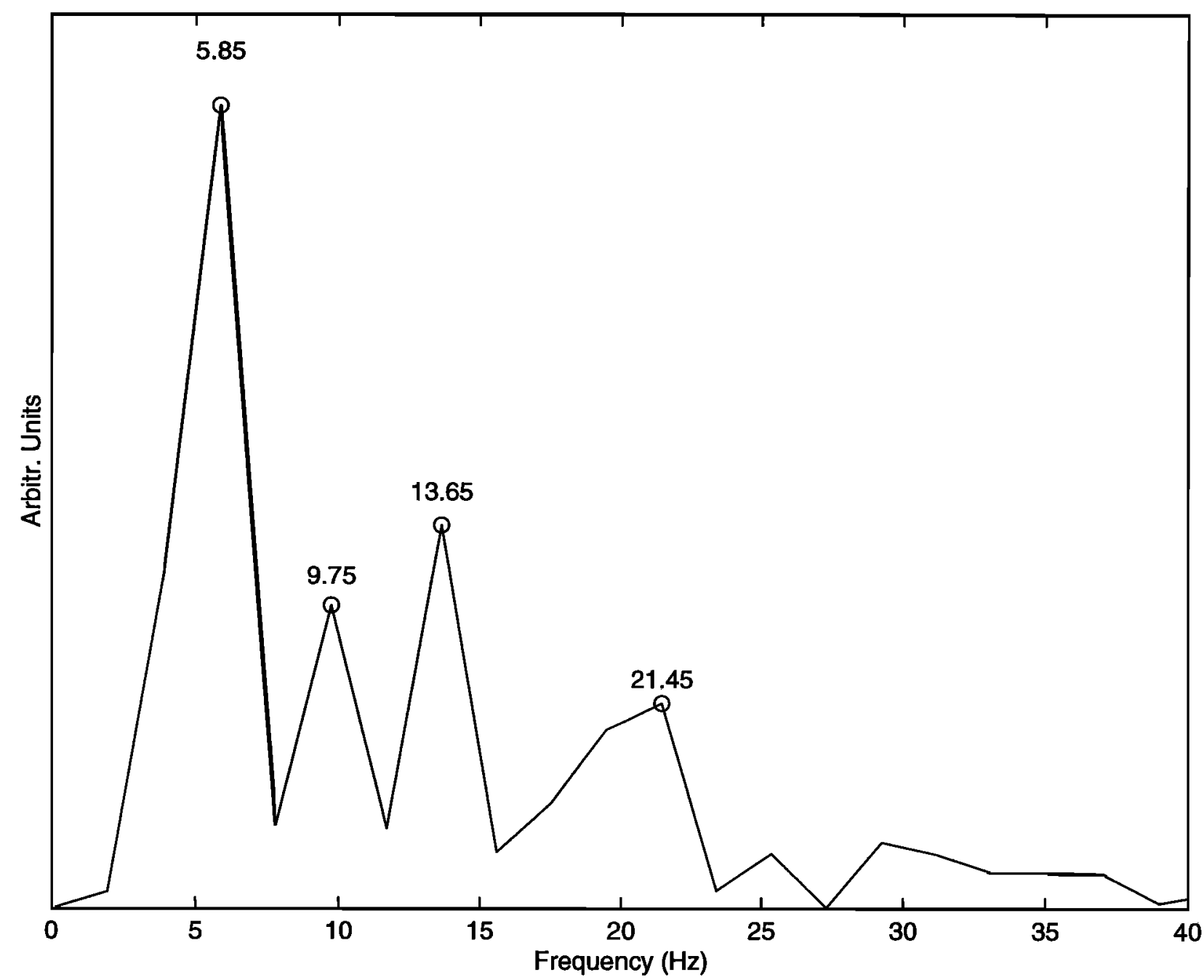

Figure 4. Power spectrum of $\delta N_{e} / N_{e}$ for the second time interval (selection 2), within a density enhancement.

performed. Clear results are obtained when selecting (i.e., filtering) the autobispectra values for which the bicoherency is greater than the threshold 0.6. This is the case for the density fluctuations but not for the electric field fluctuations (where the maximum of the bicoherency function is below 0.5$)$. The filtered bispectra of $\delta N_{e}(t) / N_{e}(t)$ are displayed in Plate 2. They present the same peaks as in Plate 1 (top rectangles). Curiously, they involve frequencies that are seen on the $E_{h}$ autopower spectra $(7.8$ and $15.6 \mathrm{~Hz})$ but not on the $\delta N_{e}(t) / N_{e}(t)$ autopower spectra. This suggests that there is a cross nonlinear interaction first, then that the phase relationships are duplicated (via Maxwell's equations) to the density fluctuations and, with a weaker efficiency, to the electric field.

In parallel to Figure 3, the temporal evolution of the cross-bispectrum maximum value and of the crossbispectra value obtained for the frequency couple (7.8, $5.85 \mathrm{~Hz}$ ) is displayed in Figure 5. The filtered electric field $E_{h}$ and the plasma frequency are recalled in the two bottom panels. The time step is 1282 samples $(0.25 \mathrm{~s})$. At every time step, the bispectrum is estimated using one FFT performed over 2564 samples $(0.5 \mathrm{~s})$. It clearly appears, first, that the nonlinearities are concentrated within positive density gradients, and second, that the coupling between 7.8 and $5.85 \mathrm{~Hz}$ is often the strongest.

\section{Discussion}

Spectral analysis performed by Cerisier et al. [1985], on electric and density fluctuations observed in the $F$ region of the ionosphere by the Aureol-3 satellite, have been remade with a higher-frequency resolution. They have been completed by estimations of bispectra and bicoherency functions. The results of the new analysis can be summarized as follows.

The power spectra of the electric field components present several frequency peaks. Three are relatively well defined: the first at $7.8 \mathrm{~Hz}$, a second around 15.6 

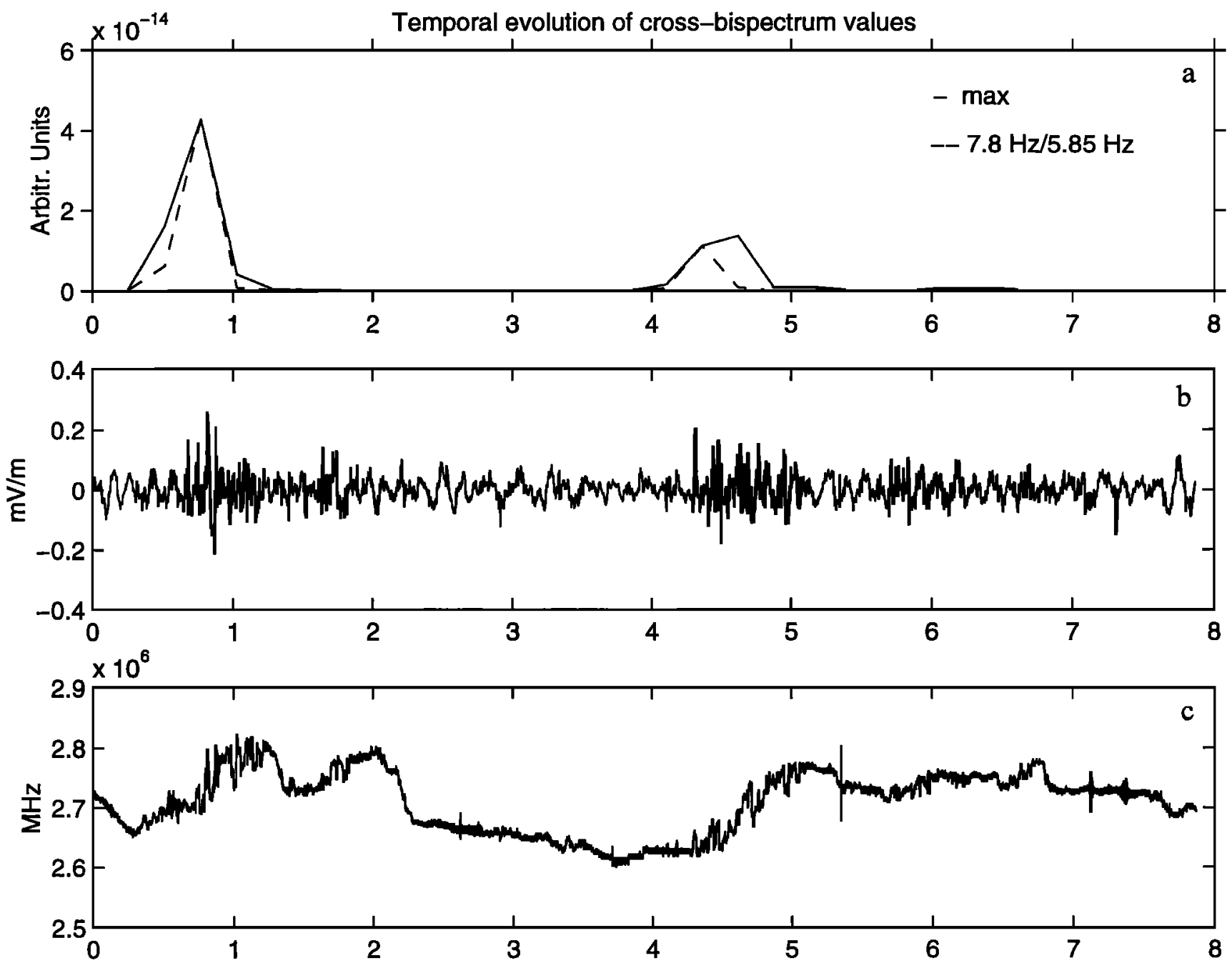

Figure 5. (a) Time variation over the full time interval (selection $1+$ selection 2) of the maximum value of the autobispectra and of the value of the auto bispectra at $f_{m}=7.8 \mathrm{~Hz}$ and $f_{n}=5.85 \mathrm{~Hz}$; (b) the electric field fluctuation and (c) the plasma density over the same time interval.

$\mathrm{Hz}$, and a third between 20 and $24 \mathrm{~Hz}$. Time-dependent peaks may also be seen. According to the power of the electric field, the expected value of the refractive indexes and the sensitivity of the magnetic sensors, it is impossible to decide whether the emissions associated with the lowest-frequency peaks are electrostatic or electromagnetic.

The power spectra of the density fluctuations have three relatively stable frequency peaks: one at 5.85 $\mathrm{Hz}$, the second at 13.65 , and the third at $17.55 \mathrm{~Hz}$. Time-dependent peaks are seen at 9.75 and $21.45 \mathrm{~Hz}$. One notes that the $13.65-\mathrm{Hz}$ frequency in $\delta N_{e}(t) / N_{e}(t)$ may be obtained by adding the $5.85-\mathrm{Hz}$ frequency in $\delta N_{e}(t) / N_{e}(t)$ to the $7.8-\mathrm{Hz}$ in $E_{h}(t)$. In the same way, the $21.45-\mathrm{Hz}$ frequency in $\delta N_{e}(t) / N_{e}(t)$ may be obtained by adding the $5.85-\mathrm{Hz}$ frequency in $\delta N_{e}(t) / N_{e}(t)$ to the $15.6-\mathrm{Hz}$ in $E_{h}(t)$. For the sake of simplicity, relationships involving negative frequencies are not discussed here.
Values above 0.6 of the cross bicoherency functions show that phase relationships exist between the density and electric field fluctuations for these two sets of coupled frequencies. According to the values of the cross bispectra at the same frequencies, one may consider that some energy is transferred between the frequencies (or wavelengths) and the fluctuations. Values above 0.6 of the $\delta N_{e}(t) / N_{e}(t)$ autobicoherency function, for the same sets of frequencies, suggest that the cross interaction between frequencies is duplicated (via Maxwell's equations) on the density fluctuations. The same phenomenon is less clear on the field fluctuations.

These features raise at least four questions: (1) what is the origin of the peaks at 7.8 and $15.6 \mathrm{~Hz}$ on the electric fluctuations? (2) what is the origin of the peak at $5.85 \mathrm{~Hz}$ in the density fluctuations? (3) what is the mechanism responsible for the energy transfers between the frequencies and the fluctuations? and (4) what is the importance of that phenomenon in the plasma 
structuring of the ionosphere? According to the data we have in hand, we can only suggest answers for the two first questions and give indications of research for the third. Answers to question four are left for future work.

Let consider the first question. Although ULF waves observed on satellites may be of instrumental origin we have no reason to suspect a similar effect for the 7.8 and $15.6-\mathrm{Hz}$ peaks on the electric fluctuations. Reports on ULF waves of instrumental origin usually refer to a rectification of a powerful signal produced by a Langmuir wave or an upper hybrid resonance [see, e.g., Stasiewicz et al., 1974]. However, no wave emissions are seen on the HF ISOPROBE antennas [Béghin et al., 1982] at the time of the observation. Now, the absence of $\mathrm{HF}$ emissions also rules out any possible natural parametric instability driven by a Langmuir wave or an upper hybrid resonance as described, e.g., by Tripathi and Sharma [1988]. Obviously, the 7.8 and $15.6 \mathrm{~Hz}$ may be produced locally as electrostatic emissions. However, any geophysicist observing a $7.8-\mathrm{Hz}$ frequency in the ionosphere is tempted to associate it with an electromagnetic emission associated with Schumann resonances [Sentman, 1995]. It has been known for long that electromagnetic waves generated during atmospheric storms are trapped within the Earth/ionosphere waveguide and resonate at $\sim 7.8 \mathrm{~Hz}$ and its spherical harmonics. A priori, there is no way to transmit a significant amount of wave energy density at the Aureol-3 altitude. However propagation models taking into account the existence of strong density gradients have not been investigated so far. Even if it is weak, the probability of observing Schumann resonances in the upper $F$ region of the ionosphere is nonnull. One is faced with similar problems to interpret the observations of fractional-hop whistlers coming from the Earth surface and reaching Aureol-3 in a frequency range going from a few hertz to $500 \mathrm{~Hz}$. Although the classical ray tracings do not predict them, the observations are made.

As far as the density fluctuations are concerned, it seems reasonable to consider that, as for the frequency peaks in the electric field fluctuations, the $5.85-\mathrm{Hz}$ frequency is of a natural origin. The simplest hypothesis is that it is produced by crossings of $\sim 1 \mathrm{~km}$ density structures. However, there is no way to check that hypothesis.

Now, whatever the nature of the wave emissions at 7.8 and $15.6 \mathrm{~Hz}$ (electrostatic or electromagnetic) and the origin of the density structure at $5.85 \mathrm{~Hz}$, both observations are the result or the signature of instability process(es). Assuming an electrostatic wave field and considering (1) the conditions of observation of the field fluctuations on Aureol-3 (stability of one side of field-aligned plasma density enhancements and instability of the other side) and (2) the very similar values they obtained for the spectral indices of the density and electric fluctuations, Cerisier et al. [1985] claimed that the instability was of the gradient drift type. Although this instability assumes a background electric field parallel to the density gradient [Kintner and Seyler, 1995], which is not obvious at the Aureol-3 altitude, this conclusion was not contested by authors who worked on the same data [Tsunoda, 1988] or on comparable data [Basu et al., 1990]. Nevertheless, Basu et al. [1990] presented evidences showing that consideration on the spectral shape were not enough for an unambiguous determination of an instability type. They showed that the magnitude $\Delta E$ of the electric field perturbation that is associated with $\delta N_{e}(t) / N_{e}(t)$ could give a supplementary constraint. However, it is difficult to know if the relation they got, from statistical studies of two types of instability, can be applied to the Aureol-3 data. If it is the case, it is likely that the instability we are looking for is not of the gradient drift type. Now, testing an instability model on real data being a very delicate task let assume that the instability we are studying is the gradient drift. In that case, according to Kintner and Seyler [1985] the instability could evolve towards a three or four wave nonlinear process, which could explain the results of our bispectral analyses.

Other instabilities may produce three or four wave interactions. They can be superposed to an instability of the gradient drift type. A full review is beyond the scope of the paper. However, the analyses being made in an inhomogeneous medium one cannot rule out the triggering of drift waves, i.e., of unstable magnetoionic modes [Mikhailovsky, 1983; Stix and Swanson, 1983] by the wave at $7.8 \mathrm{~Hz}$. It is likely in that case that the unstable modes are electrostatic. However, the triggering wave may be electrostatic or electromagnetic.

As a matter of fact, the phase coupling pointed out on the cross-bispectra and bicoherency functions between waves at 7.8 and $15.6 \mathrm{~Hz}$ in one hand, and density fluctuations at $5.85 \mathrm{~Hz}$ in the other hand suggest the existence of a parametric instability or of a coupling between two modes [Chen, 1974; Oraevsky, 1984; Mima and Nishikawa, 1984]. However, in the two cases (parametric instability or mode coupling) the difficulty is to find the driver. Examples of nonlinear interactions driven by natural resonances such as Langmuir wave, upper hybrid wave and lower hybrid wave, and attributed to a parametric instability can be found in the literature [Lee and Kuo, 1981; Riggin and Kelley, 1982; Tripathi and Sharma, 1988; Stasiewicz et al., 1996]. Other examples involving VLF transmitter signals have been published [Tanaka et al., 1987; Lagoutte et al., 1989; Trakhtengerts and Hayakawa, 1993]. One of them has been interpreted by Sotnikov et al. [1991] as a coupling between a VLF transmitter signal and a natural emission. However, in the case we are interested in, where the wave frequencies are well below any natural resonance and where there is apparently no powerful source of energy transmitted from the ground, it does not seem possible to invoke such types of instability.

Now, examining the power spectra of the Lagoutte 
et al. [1989] Aureol-3 data on nonlinear interaction triggered by a VLF transmitter signal, one observes that the wave which is supposed to trigger the interaction (i.e., the transmitted emission) is not the one whose the wave energy density is maximum. This suggests that the quantity to take into account in such interaction is not the wave energy density at a given point but the flux of energy conveyed within the interaction region. Then, the size of the interaction region and the group velocity of the wave within that region are probably more important parameters than the wave energy density at the observation point. It turns out that even if it is observed with a weak energy density, the upgoing propagating part of Schumann resonances may trigger a parametric instability or a mode coupling involving a density fluctuation at $\sim 5.85 \mathrm{~Hz}$. In both cases, the wave reaching the interaction region has obviously to be electromagnetic, even if the propagation characteristics are such that the electric field is largely dominant. However, before exploring in details such directions of research, one must try to find other cases and to make statistical estimations of all the relevant wave and plasma parameters. This supposes an extensive investigation of the Aureol-3 satellite data that are the only ones to contain simultaneous measurements of the field and density fluctuations. Such a work is in progress.

Acknowledgments. The authors thank the referees for very constructive comments. LPCE is an INSU laboratory which has a convention with the University of Orléans. Partially funded by the French Embassy in Sweden under the authority of Dr. Guastavino, Scientific Counselor.

Michel Blanc thanks Sasha Kustov and Jean-Pierre Saint-Maurice for their assistance in evaluating this paper.

\section{References}

Allis, W. P., S. J. Buchsbaum, and A. Bers, Waves in Anisotropic Plasma, MIT Press, Cambridge, Mass., 1963.

Basu, Su., S. Basu, E. Mackenzie, P. F. Fougere, W. R. Coley, N. C. Maynard, J. D. Winningham, M. Sugiura, W. B. Hanson, and W. R. Hoegy, Plasma structuring by the gradient drift instability at high latitudes and comparison with velocity shear driven processes, J. Geophys. Res., 93, 115, 1988.

Basu, Su., S. Basu, E. Mackenzie, W. R. Coley, J. R. Sharber, and W. R. Hoegy, Plasma structuring by the gradient drift instability at high latitudes and comparison with velocity shear driven processes, J. Geophys. Res., 95, 7799, 1990.

Béghin, C., J. F. Karczewski, B. Poirier, R. Debrie, and N. Massewitch, The ARCAD-3 ISOPROBE experiment for high time resolution thermal plasma measurements, Ann. Geophys., 38, 615, 1982.

Béghin, C., J. C. Cerisier, J. L. Rauch, J. J. Berthelier, F. Lefeuvre, R. Debrie, O. A. Molchanov, O. A. Maltseva, and N. I. Massevitch, Experimental evidence of plasma ducts in the ionospheric trough and in the auroral zone, Adv. Space Res., 5(4), 229, 1985.

Berthelier, J. J., et al., Measurements of the VLF electric and magnetic components of waves and DC electric field on-board the AUREOL 3 satellite: The TBF-ONCH experiment, Ann. Geophys., 38(5), 643, 1982.
Bosqued, J. M., H. Barthe, J. Coutelier, J. Crasnier, J. Cuvilo, J. L. Medale, H. Reme, J. A. Sauvaud, and R. A. Kovrazkine, The low-energy electron and ion spectrometer on the AUREOL 3 satellite: The SPECTRO experiment, Ann. Geophys., 38(5), 567, 1982.

Cerisier, J. C., J. J. Berthelier, and C. Béghin, Unstable density gradients in the high-latitude ionosphere, Radio Sci., 20, 755, 1985.

Chen, F. F., Introduction to plasma physics, Plenum, New York, 1974.

Daubechies, I., Ten lectures on wavelets, CBNS-NSF Regional Conference Series in Applied Mathematics, Philadelphia, 1992.

Dudok de Wit, T., and V. V. Krasnosel'skikh, Wavelet bicoherence analysis of strong plasma turbulence at the Earth's quasi-parallel bowshock, Phys. Plasma, 2, 262, 273, 1995.

Hinich, M. J., Detecting a transient signal by bispectral analysis, IEEE Trans. Acoust. Speech Signal Processing, $38,1277,1990$.

Kersley, L., F. E. Pryse, and N. S. Wheadon, Small-scale irregularities associated with a high-latitude electron density gradient: Scintillation and EISCAT observations, $J$. Atmos. 'Terr. Phys., 50, 557, 1988.

Kim, K. I., and E. J. Powers, Digital bispectral analysis and its applications to nonlinear wave interactions, IEEE. Trans. Plasma Sci., PS-7(2), 120, 1979.

Kintner, P. M., and C. E. Seyler, The status of observations and theory of high latitude ionospheric and magnetospheric plasma turbulence, Space Sci. Res., 41, 91, 1985.

Lagoutte, D., F. Lefeuvre, and J. Hanasz, Application of bicoherence analysis in study of wave interactions in space plasma, J. Geophys. Res., 94, 435, 1989.

Lee, M. C., and S. P. Kuo, Production of lower hybrid waves and field-aligned plasma density striation by whistlers, $J$. Geophys. Res., 86, 873, 1981.

Linson, L. M., and J. B. Workman, Formation of striations in ionospheric plasma clouds, J. Geophys. Res., 75, 3211, 1970.

Maggs, J. E., and G. J. Morales, Magnetic fluctuations with field-aligned striations, Geophys. Res. Lett., 23, 633, 1996.

Marple, S. L. Digital Spectral Analysis With Applications, Prentice Hall, Englewood Cliffs, N. J, 1987.

Mikhailovsky, A. B., Instabilities in inhomogeneous plasma, in Handbook of Plasma Physics, Basic Plasma Physics I, edited by A. A. Galeev and R. N. Sudan, p.587, NorthHolland, New York, 1983.

Mima, K., and K. Nishikawa, Parametric instabilities and wave dissipation, in Handbook of Plasma Physics, Basic Plasma Physics II, edited by N. M. Rosemblath and R. Z. Sagdeev, p.451, North-Holland, New York, 1984.

Muldrew, D. B., and J. F. Vickrey, High-latitude F region irregularities observed simultaneously with ISIS 1 and the Chatanika radar, J. Geophys. Res., 87, 907, 1982.

Nikias, C. L., and A. P. Petropulu, Higher Order Spectra Analysis: A Nonlinear Signal Processing Framework, signal processing ser., edited by A. V. Oppenheim, Prentice Hall, Englewood Cliffs, N. J., 1993.

Oraevsky, V. N., Parametric instabilities in magnetospheric plasmas, in Handbook of Plasma Physics, Basic Plasma Physics II, edited by N. M. Rosemblath and R. Z. Sagdeev, p.38, North-Holland, New York, 1984.

Orringer, O., Frontal analysis program, Rep. ASRL TR 1023, Aeroelastic and Struct. Lab., Mass. Inst. of Technol., Cambridge, 1974.

Pflug, A., G. Ioup, W. Ioup, and R. Field, Prediction of signal-to-noise ratio gain for passive higher order correla- 
tion detection of energy transients, J. Acoust. Soc. Am., 98(1), 249, 1995.

Rezeau, L., G. Belmont, B. Gueret, and B. Lembege, Crossbispectral analysis of the electromagnetic field in a beamplasma interaction, J. Geophys. Res., 102, 24,387, 1997.

Riggin, D., and M.C. Kelley, The possible production of lower hybrid parametric instabilities by VLF ground based transmitters and by natural emissions, J. Geophys. Res., $87,2545,1982$.

Rino, C. L., R. C. Livingston, R. T. Tsunoda, R. M. Robinson, J. F. Vickrey, C. Senior, M. D. Cousins, J. Owen, and J. A. Klobuchr, Recent studies of the structure and morphology of auroral zone F region irregularities, Radio Sci., 18,1167, 1983.

Sentman, D. D., Schumann resonances, in CRC Handbook of Atmospheric Electrodynamics I, edited by H. Volland, p. 267, CRC Press, Boca Raton, Fla., 1995.

Sotnikov, V. I., V. Fiala, F. Lefeuvre, and D. Lagoutte, Excitation of sidebands due to nonlinear coupling between a VLF transmitter signal and natural ELF emission, $J$. Geophys. Res., 96, 11363, 1991.

Stasiewicz, K., B. Holback, V. V. Krasnosselskikh, M. Boehm, R. Bostrom, and P. M. Kintner, Parametric instabilities of Langmuir waves observed by Freja, J. Geophys. Res., $10121,515,1996$.

Stix, T. H., Waves in Plasmas, American Institute of Physics, New York, 1992.

Stix, T. H., and D. G. Swanson, Propagation and modeconversion for waves in nonuniform plasmas, in Handbook of Plasma Physics, Basic Plasma Physics I, edited by A. A. Galeev and R. N. Sudan, p. 335, North-Holland, New York, 1983.

Tanaka, Y., D. Lagoutte, M. Hayakawa, F. Lefeuvre, and S. Tajima, Spectral broadening of VLF transmitter signals and sideband structure observed on AUREOL-3 satellite at middle latitudes, J. Geophys. Res., 92, 7551, 1987.

Trakhtengerts, V. Y., and M. Hayakawa, A wave interaction in whistler frequency range in space plasma, J. Geophys. Res., 98, 19,205, 1993.

Tripathi, Y. K., and R. P. Sharma, Some parametric instabilities of an ordinary electromagnetic wave in magnetized plasmas, Phys. Rev. A, 38, 2991, 1988.
Tsunoda, R. T., High- latitude F region irregularities: A review and synthesis, Rev. Geophys., 26, 719, 1988.

Vickrey, J. F., C. L. Rino, and T. A. Potemra, Chatanika/Triad observations of unstable ionization enhancements in the auroral F region, Geophys. Res. Lett., 7, 789, 1980.

Villain, J. P., C. Béghin, and C. Hanuise, ARCAD-3 SAFARII coordinated study of auroral and polar $F$ region ionospheric irregularities, Ann. Geophys., 4, 61, 1986.

Weber, E. J., J. Buchau, J. G. Moore, J. R. Sharber, R. C. Livingston, J. D. Winningham, and B. W. Reinisch, $\mathrm{F}$ layer ionisation patches in the polar cap, J. Geophys. Res., 89, 1683, 1984.

Weber, E. J., R. T. Tsunoda, J. Buchau, R. E. Sheehan, D. J. Strickland, W. Whiting, and J. G. Moore, Coordinated measurements of auroral zone plasma enhancements, $J$. Geophys. Res., 90, 6497, 1985.

Weber, E. J., J. A. Klobuchar, J. Buchau, H. C. Carlson Jr., R. C. Livingston, O. de la Beaujardiere, M. McCready, J. G. Moore, and G. J. Bishop, Polar cap $F$ layer patches: Structure and dynamics, J. Geophys. Res., 91, 12,121, 1986.

Welch, P. D., The use of fast Fourier transform for the estimation of power spectra: A method based on time averaging over short, modified periodograms, IEEE Trans. Audio Electroacoust., $A U-15,70,1967$.

Yampolski, Y. M., P. V. Bliokh, V. S. Beley, V. G. Galushko, and S. B. Kasheev, Nonlinear interaction between Schumann resonances and HF signals, J. Atmos. Solar. Terr. Phys., 59, 335, 1997.

Zhao, Z. Y., F. Lefeuvre and A. Masson, Nonlinear interactions between ELF electrostatic turbulence and irregularities in electronic density, Proceedings of the 1997 International Symposium on Radio Propagation, p. 247, Chinese Journal of Radio Science, Qingdao, China, 1997.

D. Lagoutte, F. Lefeuvre, J.-L. Rauch, and Z. Y. Zhao, Laboratoire de Physique et Chimie de l'Environnement, CNRS, 3A Avenue de la Recherche Scientifique, 45071 Orléans, France.

A. Masson, Swedish Institute of Space Physics, Uppsala, S-755 91, Sweden. (masson@irfu.se)

(Received January 20, 1998; revised February 26, 1999;

accepted April 18, 1999.) 